\title{
Methotrexate binding causes structural and functional changes in lung cystatin
}

\author{
Mohd Shahnawaz Khan, Medha Priyadarshini, Sadia Sumbul and Bilqees Bano ${ }^{\varpi}$ \\ Department of Biochemistry, Faculty of Life Sciences, Aligarh Muslim University, Aligarh, U.P., India
}

\begin{abstract}
Regulation of cysteine proteinases and their inhibitors is of utmost importance in diseases like lung cancer, chronic inflammatory conditions such as asthma, emphysema, and idiopathic pulmonary fibrosis. Proteaseantiprotease imbalance accelerates disease progression. In the present study, the effect of antineoplastic and antirheumatic drug methotrexate (MTX) on lung cystatin (a cysteine protease inhibitor) was studied to explore drug induced changes in functional and structural integrity of the protein. The basic binding interaction was studied by UV-absorption, FT-IR and fluorescence spectroscopy. The quenching of protein fluorescence confirmed the binding of MTX with goat lung cystatin (GLCI). Stern-Volmer analysis of MTX-GLC-I system at different temperatures indicates the presence of static component in the quenching mechanism. The thermodynamic parameters $\Delta H^{0}$ and $\Delta S^{0}$ were $-3.8 \mathrm{~kJ} / \mathrm{mol}$ and $94.97 \mathrm{~J} \cdot \mathrm{mol}^{-1} \cdot \mathrm{K}^{-1}$, respectively, indicating that both hydrogen bonds and hydrophobic interactions played a major role in the binding of MTX to GLC-I. Methotrexate $(7 \mu \mathrm{M})$ caused complete inactivation of lung cystatin after 6 hours. The results of FT-IR spectroscopy reflect perturbation of the goat lung cystatin on interaction with MTX. Methotrexate induced loss of function change in the inhibitor could provide a rationale for the off target tissue injury caused by the drug and for the design of agents against such an injury.
\end{abstract}

Keywords: goat lung cystatin, methotrexate, emphysema, FTIR

Received: 20 September, 2009; revised: 14 May, 2010; accepted: 03 November, 2010; available on-line: 29 November, 2010

\section{INTRODUCTION}

Cystatins, the ubiquitous non-covalent competitive inhibitors of cysteine proteinases such as cathepsins $\mathrm{B}, \mathrm{H}$, $\mathrm{L}$ and S (Ekiel et al., 1997), have myriad of biologic activities attributed to them, like proliferation, differentiation, ageing and death, and antigen presentation besides protection of cells from unwanted proteolysis (Turk et al., 2008). Cystatins also play a vital role in the homeostasis of intracellular and extracellular matrix whose recycling is important otherwise it will cause a number of diseases predominatly lung fibrosis and emphysema (Katzenstein \& Myers, 1998).

Methotrexate (4-amino-10-methylfolic acid/amethopterin, MTX) (Fig. 1), a folic acid antagonist, is recognized as one of the most important and commonly used drugs in the treatment of leukemia, lymphomas, osteosarcoma, and rheumatoid arthritis (Neves et al., 2009). Despite its indisputable efficacy and good tolerance profile, side effects of MTX vary from malaise and asthenia to pneu- monitis or pancytopenia, which can be fatal (Hughes et al., 1987; Neves et al., 2009).

A number of slightly different conformational states are available to proteins which differ drastically in their function. The marginal stability of the native conformation is also a delicate balance of various interactions in proteins (van der Waals, electrostatic, hydrogen bonds and disulphide bridges) (Sneppen \& Zocchi, 2005) which is affected by $\mathrm{pH}$, temperature or addition of small molecules such as substrates, coenzymes, inhibitors and activators that bind especially to the native state and thus alter this fragile equilibrium. In this regard, most drugs exert their activity by interaction with proteins.

Drug accumulation at off-target sites in the body leads to unintended adverse reactions (Taniguchi et al., 2007; Scheiber et al., 2009) and drug/ligand induced protein structure conformational alterations (Takeda et al., 1988) are prime problems complicating drug medical therapy by affecting the cellular proteins.

Keeping in view the importance of cystatins in maintenance of proper lung function, the effect of methotrexate on the integrity of a newly purified cysteine protease inhibitor from Capra hircus lung was explored. We have already reported the purification and characterization of goat lung cystatin (GLC) identified to be a cystatin superfamily variant based on sequence homology (Khan \& Bano, 2009). GLC is a single subunit $63 \mathrm{kDa}$ protein, possessing $48.8 \% \quad \alpha$-helix and substantial amount of $\beta$ structure. Any conformational modulation induced upon methotrexate interaction leading to alterations in the inhibitor's function could be extended to the drug's lung damaging effects. Thus, CD, fluorescence and FTIR spectroscopy were employed to assess any structural changes in lung cystatin on interaction with methotrexate. Functional changes were analyzed by assessment of the inhibitory potential of the drug-bound protein.

\section{MATERIALS AND METHODS}

Materials. Goat lung cystatin was purified in our lab and methotrexate (solution for injection) was from PlivaLachema a.s.

Purification of thiol protease inhibitor from goat lung. Cysteine protease inhibitor was isolated and purified from goat lung in high yield and fold purification by a simple two step procedure, ammonium sulphate precipitation and ion exchange chromatography (Khan \& Bano, 2009).

e-mail: banobilqees@gmail.com

Abbreviations: FT-IR, Fourier transform-infra red spectroscopy; GLC-I, goat lung cystatin I; MTX, methotrexate, 4-amino-10-methylfolic acid/amethopterin 
Drug-protein interactions. Goat lung cystatin concentration was fixed at $1 \mu \mathrm{M}$ and the drug (MTX) concentration was varied from $0.25 \mu \mathrm{M}$ to $7 \mu \mathrm{M}$. Final volume $(3 \mathrm{ml})$ of sample was made up with by $0.05 \mathrm{M}$ sodium phosphate buffer $\mathrm{pH} 7.4$ and the reaction mixture was incubated for $2 \mathrm{~h}$ before the fluorescence spectra were taken. Fluorescence spectra were recorded at three different temperatures (298, 308, and 318 K). Fluorescence measurements were carried out on a Shimadzu spectrofluorimeter model RF-540 equipped with a data recorder DR-3. The fluorescence was recorded at 300$400 \mathrm{~nm}$ after exciting the protein solution at $280 \mathrm{~nm}$ for total protein fluorescence. The slits were set at $5 \mathrm{~nm}$ for excitation and emission. The path length of the sample was $1 \mathrm{~cm}$.

Absorbance spectra. The UV measurements of GLC in the presence and absence of MTX were made in the range of 200-300 nm. Absorbance spectra were recorded on a double beam Shimadzu UV-Vis spectrophotometer UV-1700 using a cuvette of $1 \mathrm{~cm}$ path length.

Activity measurements of goat lung cystatin in the presence of MTX. The inhibitory activity of purified GLC was assessed by its ability to inhibit caseinolytic activity of papain by the method of Kunitz (1947). GLC was incubated with increasing concentrations of (MTX) (from $0.25 \mu \mathrm{M}$ to $7 \mu \mathrm{M}$ ) at $25^{\circ} \mathrm{C}$ for different time intervals $(2-6 \mathrm{~h})$ before its inhibitory activity was measured.

Fourier transform infrared (FT-IR) spectroscopy. FT-IR measurements were made at room temperature on a Nicolet Nexus 670 FT-IR spectrometer equipped with a germanium attenuated total reflection (ATR) accessory, a DTGS KBr detector and a $\mathrm{KBr}$ beam splitter. All spectra were taken via the attenuated total reflection (ATR) method with resolution of $4 \mathrm{~cm}^{-1}$ and 10 scans. Spectra of buffer and GLC solution were collected at the same condition. The absorbance of buffer solution (0.05 M, Na-P buffer, $\mathrm{pH}$ 7.5) was subtracted from that of GLC solution. The spectra were taken in the region $1800-1400 \mathrm{~cm}^{-1}$ at physiological $\mathrm{pH}$.

Statistical analysis. All the experiments were conducted in four replicates. All data are expressed as mean \pm SEM. Two-way analysis of variance (ANOVA) of data was performed to check for significant differences between MTX concentrations and incubation times. The calculated $\mathrm{F}$ values of the test were compared with the obtained values from the Fisher-Snedecor table. Two-way ANOVA was finished using SPSS 10.0 software. $P<0.05$ was taken as statistically significant.

\section{RESULTS}

\section{Drug-protein interactions}

The fluorescence spectra of GLC-I in the presence of different concentrations of MTX were recorded in the range of 300-400 $\mathrm{nm}$ upon excitation at $280 \mathrm{~nm}$. MTX caused quenching of the intrinsic fluorescence of GLC-I (Fig. 2) accompanied by a red shift $(11 \mathrm{~nm})$ of maximum fluorescence at $7 \mu \mathrm{M}$. These results indicated interactions between MTX and GLC-I resulting in a non-fluorescent complex. The fluorescence quenching data was analysed by the Stern-Volmer equation:

$\mathrm{F}_{0} / \mathrm{F}=1+K_{\mathrm{SV}}[\mathrm{Q}]$

where $\mathrm{F}_{0}$ and $\mathrm{F}$ are steady-state fluorescence intensities in the absence and presence of quencher, respectively,<smiles>CN(Cc1cnc2nc(N)nc(N)c2c1)c1ccc(C(=O)N(CCC(=O)O)C(=O)OCCC(=O)O)cc1</smiles>

Figure 1. Chemical structure of methotrexate, an antineoplastic and antirheumatic drug

$K_{\mathrm{SV}}$ the Stern-Volmer quenching constant and [Q] is the concentration of quencher (Fig. 3). The $K_{\mathrm{SV}}$ values obtained at three different temperatures are shown in Table 1.

\section{Determination of binding constant $(K)$ and number of binding sites $(\mathrm{n})$}

When small molecules bind independently to a set of equivalent sites on a macromolecule, the equilibrium between free and bound molecules is given by the equation (Feng et al., 1998; Gao et al., 2004):

$\log \left[\left(\mathrm{F}_{0}-\mathrm{F}\right) / \mathrm{F}\right]=\log K+\mathrm{n} \log [\mathrm{Q}]$

where $K$ and $\mathrm{n}$ are the binding constant and the number of binding sites, respectively. Thus, a plot of $\log \left(\mathrm{F}_{0}-\right.$ $\mathrm{F}) / \mathrm{F}$ versus $[\mathrm{Q}]$ can be used to determine $K$ as well as $\mathrm{n}$. The values of $K$ and $n$ obtained at three different temperatures are shown in Table 1.

\section{Type of interaction force between MTX and GLC-I}

In order to identify the interaction force of MTX with GLC-I, the thermodynamic parameters, i.e., free energy changes $\left(\Delta G^{0}\right)$, enthalpy changes $\left(\Delta H^{0}\right)$, and entropy changes $\left(\Delta S^{0}\right)$ of the interactions were calculated from the following equations:

$\ln K=-\Delta H^{0} / R T+\Delta S^{0} / R$

$\Delta G^{0}=-\mathrm{R} T \ln K=-\Delta H^{0}-T \Delta S$

The results obtained are shown in Table 1. There was a positive entropy change while negative values were obtained for free energy and enthalpy changes.

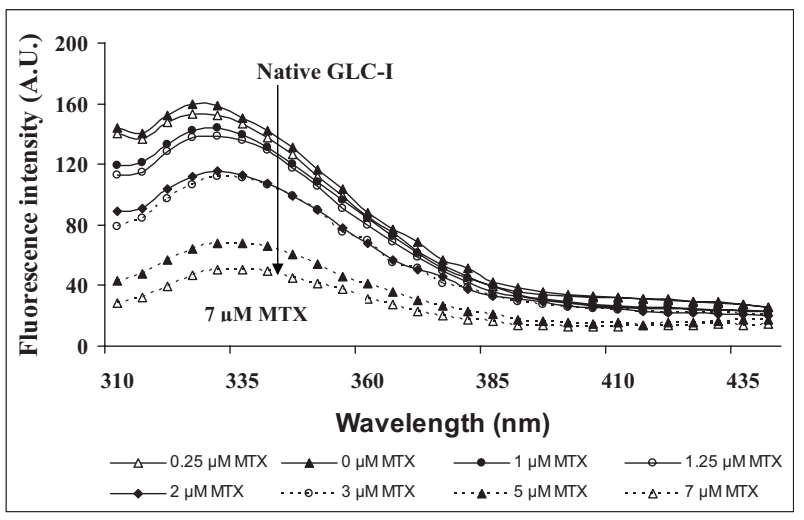

Figure 2. Fluorescence emission spectra of GLC-I in phosphate buffer, $\mathrm{pH} 7.4$, in absence and presence of MTX at excitation wavelength of $280 \mathrm{~nm}$ 


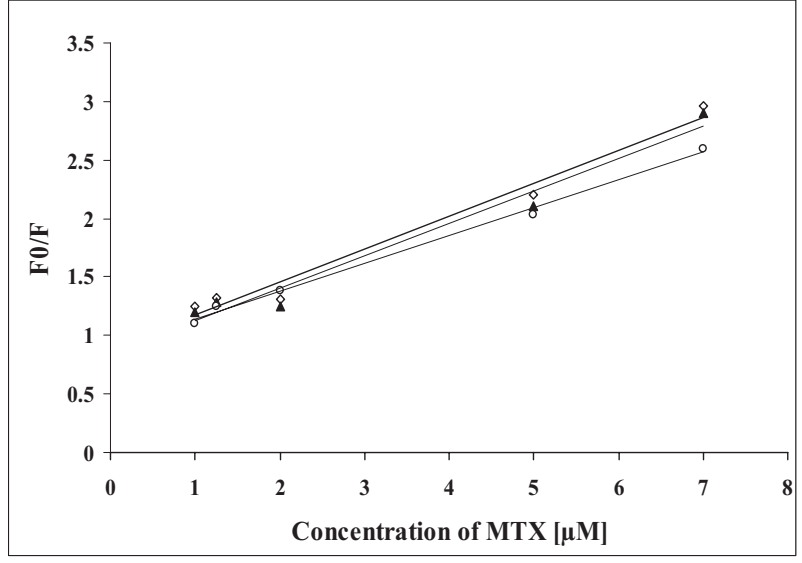

Figure 3. Stern-Volmer plot for the binding of GLC-I to MTX at different temperatures. The symbol $(\Delta)$ denote $298 \mathrm{~K},(\diamond) 308$ $\mathrm{K}$ and $(\mathrm{O}) 318 \mathrm{~K}$

\section{UV-Vis absorbance studies}

The interaction between MTX and GLC-I was studied from UV-vis absorbance spectral data (Fig. 4). The UV absorbance intensity of GLC-I increased with MTX concentration. The addition of the drug results in a distinct shift of MTX-GLC-I spectrum toward longer wavelengths (red shift).

\section{Inhibitory activity of GLC-I in the presence of MTX}

Changes in the inhibitory activity of GLC-I after incubation for different times (2-6 h) with increasing concentrations of MTX are shown in Table 2. GLC-I lost a significant fraction of its inhibitory activity at $7 \mu \mathrm{M}$ concentration of MTX after $6 \mathrm{~h}$ of incubation. The obtained data also indicates that inactivation of goat lung cystatin by MTX is concentration as well as time dependent.

\section{FT-IR measurements}

Additional evidence regarding the MTX-GLC-I interaction comes from FT-IR spectroscopy, showing drug-protein complexes. Infrared spectra of proteins exhibit a number of amide bands which represent different vibrations of the peptide moiety. This vibration

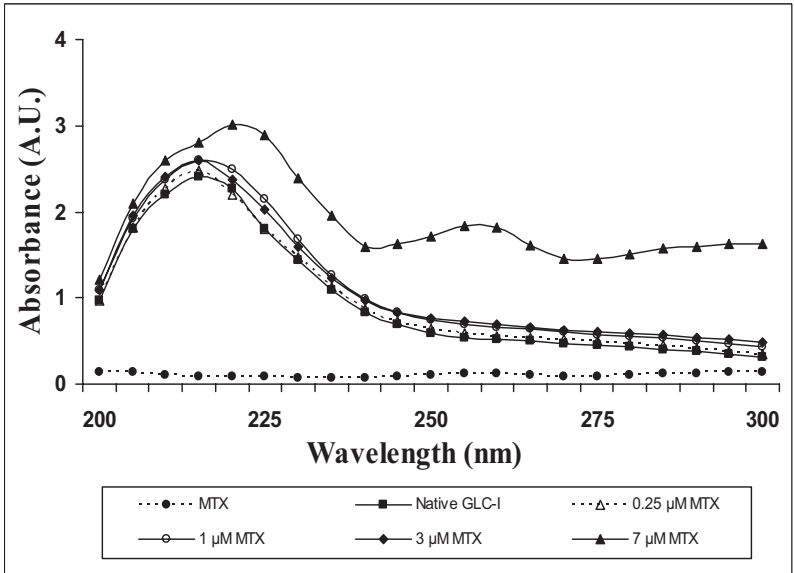

Figure 4. Absorbance spectra of GLC-I in absence and presence of MTX
Table 1. Thermodynamic parameters of MTX-GLC-I interactions at different temperature

\begin{tabular}{lccc}
\hline $\begin{array}{l}\text { Para- } \\
\text { meters }\end{array}$ & $298 \mathrm{~K}$ & $308 \mathrm{~K}$ & $318 \mathrm{~K}$ \\
\hline$K_{\mathrm{sv}}\left(\mathrm{M}^{-1}\right)$ & $2.8 \times 10^{5} \pm 0.04$ & $2.6 \times 10^{5} \pm 0.03$ & $2.3 \times 10^{5} \pm 0.04$ \\
$K\left(\mathrm{M}^{-1}\right)$ & $4.26 \times 10^{5} \pm 0.02$ & $4.0 \times 10^{5} \pm 0.042$ & $3.82 \times 10^{5} \pm 0.05$ \\
$\mathrm{n}$ & 1.11 & 0.98 & 0.97 \\
$\Delta G^{0}$ & -32.0 & -33.0 & -33.9 \\
$\mathrm{kJmol}^{-1}$ & & & \\
$\Delta H^{0}$ & & -3.8 & \\
$\mathrm{kJmol}-1)$ & & & \\
$\Delta S^{0}$ & & 94.97 & \\
$\mathrm{~J} \mathrm{~mol}^{-1} \mathrm{~K}^{-1}$ & & & \\
\hline
\end{tabular}

$K_{\mathrm{s} y}$, Stern-Volmer constant; $\Delta G^{0}$, Standard free energy change; $K$, Binding constant; $\Delta H^{0}$, Standard enthalpy change; $n$, Number of binding constant; $\Delta S^{\circ}$, Standard entropy change

mode originates from the $\mathrm{C}=\mathrm{O}$ stretching vibration of the amide group (coupled to the in-phase bending of the $\mathrm{N}-\mathrm{H}$ bond and the stretching of the $\mathrm{C}-\mathrm{N}$ bond) and gives rise to infrared bands in the region between approximately 1600 and $1700 \mathrm{~cm}^{-1}$ (Surewicz et al., 1993). The protein amide bands have a relationship with the secondary structure. Figure 5 shows FTIR spectra of the MTX free and MTX-bound form of GLC-I corrected for the absorbance of phosphate buffer. The evident peak shift of amide I band from 1652.4 to $1635.4 \mathrm{~cm}^{-1}$ indicates that the secondary structure of GLC-I is changed when MTX is added.

\section{DISCUSSION}

The estimation of pharmacokinetics and pharmacodynamics of drugs used in the leukaemia multidrug therapy is of great importance. The multidrug therapy, despite its high effectiveness, carries a risk of side effects especially of the free fractions of drugs. Thus, the present study was designed to explore the side effects of methotrexate; an antineoplastic and antirheumatic drug on the structure and functioning of cysteine protease inhibitor isolated from goat lung.

The role of cysteine proteinases in lung diseases like COPD, emphysema, lung necrosis, etc. has not been de-

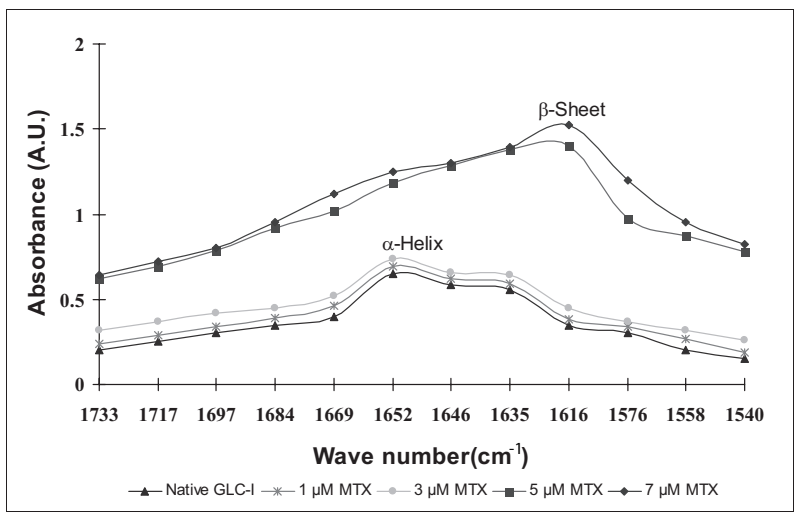

Figure 5. FT-IR spectrum of GLC-I in absence and presence of MTX

The concentration of GLC-I used was $1 \mu \mathrm{M}$ while MTX varied from $0.25 \mu \mathrm{M}$ to $7 \mu \mathrm{M}$. 
Table 2. Antiproteolytic activity of GLC-I of MTX after various time of incubation with MTX

\begin{tabular}{lccc}
\hline $\begin{array}{l}\text { Concentration of MTX } \\
(\mu \mathrm{M})\end{array}$ & 2 h of incubation & $4 \mathrm{~h}$ of incubation & $6 \mathrm{~h}$ of incubation \\
\hline 0.25 & $83.1 \pm 2.2^{*}$ & $78.2 \pm 1.5^{*}$ & $68.2 \pm 2.5^{*}$ \\
1 & $79.4 \pm 1.4^{*}$ & $75.3 \pm 2.0^{*}$ & $65.4 \pm 2.2^{*}$ \\
1.25 & $76.2 \pm 2.0^{*}$ & $70.7 \pm 2.3^{*}$ & $59.5 \pm 1.5^{*}$ \\
2 & $71.2 \pm 2.5^{*}$ & $64.5 \pm 1.5^{*}$ & $53.3 \pm 2.5^{*}$ \\
3 & $63.1 \pm 2.5^{*}$ & $55.3 \pm 2.5^{*}$ & $42.7 \pm 2.0^{*}$ \\
5 & $51.5 \pm 1.9^{*}$ & $45.5 \pm 2.2^{*}$ & $28.4 \pm 1.2^{*}$ \\
7 & $42.3 \pm 1.5^{*}$ & $34.2 \pm 2.2^{*}$ & $15.2 \pm 2.2^{*}$ \\
\hline
\end{tabular}

* Means significantly different at $P<0.05$ by two way ANOVA. The inhibitory activity of the control (in absence of MTX) was taken as $100 \%$.

fined, although they do contribute to the elastolytic activity of alveolar macrophages in COPD patients (Russell et al., 2002). The pathophysiological significance of proteases in lung disease is exemplified by $\alpha_{1}$-antitrypsin deficiency: the loss of this protease-inhibitor function leads to emphysema. However, other proteases, elastase and cathepsins may also contribute to the pathogenesis of emphysema (Zheng et al., 2000) where an increased expression of cathepsins B, D, H, L and $\mathrm{S}$ is reported (Wang et al., 2000). It is known that cathepsin inhibitors markedly reduce emphysema. Therefore, the protease antiprotease balance must be retained in order to avoid COPD.

Fluorescence measurements can give some information about the binding of small molecules to proteins, such as binding number, binding sites and binding mechanism. Fluorescence intensity of a compound can be decreased or quenched by a variety of molecular interactions, such as excited-state reactions, molecular rearrangements, energy transfer, ground state complex formation and collision quenching. The quenching of goat lung cystatin (GLC-I) is accompanied by a red-shift of maximum fluorescence. This indicates an increase of the polarity of the fluorophore environment, probably due to the hydrogen bonds between MTX and the $\mathrm{NH}_{2} \mathrm{OH}$ and $\mathrm{SH}$ groups in the inhibitor (GLC-I) which stabilize the complex (Bures et al., 1990).

The interaction forces between drugs and biomolecules include hydrophobic force, electrostatic interactions, van der Waals interactions and hydrogen bonds. The negative $\Delta H^{0}$ and positive $\Delta S^{0}$ values in the present case show that both hydrogen and hydrophobic interactions play a role in the binding of MTX to GLC-I. The positive entropy change occurs because the water molecules that are arranged in an orderly fashion around the ligand and the protein acquire a more random configuration as a result of hydrophobic interactions. The negative value of $\Delta G$ and the number of binding sites (n) showed that complex formation between MTX and GLC-I occurs spontaneously and there is only one independent binding site of interaction.

An increase in absorbance intensity accompanied by red shift of the spectrum as revealed in UV-Vis spectroscopic studies also point towards complex formation and interaction between the drug and the inhibitor (Cui et al., 2004; Hu et al., 2004).

The FT-IR analysis of goat lung cystatin in the presence of methotrexate clearly indicates that the drug causes secondary structure modifications of GLC-I (am- ide I band peak shift). Consequent to the structural changes the papaininhibitory potential of GLC-I declined on interaction with MTX, suggesting changes in the environment of the crucial amino-acid residues of the protein.

It is known that in cells incubated with MTX, intracellular level of the drug in the form of polyglutamates may be high compared with the concentration of free parent drug. However, it can be speculated that the polyglutamylated and free drug would affect GLC-I to more or less the same extent. The high drug concentration used in the present work mimicked the long duration of exposure of the drug to cellular proteins, in form of polyglutamate pool. MTX-plasma protein binding would definitely affect the effective concentration of the drug. However, as polyglutamate pools of the drug would be maintained for longer duration, the effect of binding of MTX to plasma proteins could have been neglected.

It can be concluded from our study, that inflammatory conditions such as asthma, emphysema, and idiopathic pulmonary fibrosis consequent to methotrexate therapy might not only be due to increased activity/expression of cathepsins but also due to inactivation of their inhibitor (GLC-I). It provides footage to clinical studies for exact elucidation of the impact of cystatin inhibition by MTX on lung tissue.

\section{Acknowledgements}

Facilities provided by Aligarh Muslim University are thankfully acknowledged. The assistance provided by Dr. Hamid Mustafa, Jawahar Lal Nehru Medical College and Hospital, Aligarh, is gratefully acknowledged.

\section{REFERENCES}

Assfalg-Machleidt I, Jochum M, Klaubert W et al. (1998) Enzymatically active cathepsin B dissociating from its inhibitor complexes is elevated in blood plasma of patients with septic shock and some malignant tumors. Biol Chem Hoppe-Seyler 369: 263-269.

Bernstein HG, Kirschke H, Wiederander B et al. (1996) The possible place of cathepsins and cystatins in the puzzle of Alzheimer disease. Mol Chem Neuropathol 27: 225-247.

Bures L, Lichy A, Bostik J, Spundova M (1990) The use of protein as a carrier of methotrexate for experimental cancer therapy. V. Alternative method for preparation of serum albumin-methotrexate derivative. Neoplasma 37: 225-231.

Buttle DJ, Burnett D, Abrahamson M (1990) Levels of neutrophil elastase and cathepsin B activities and cystatins in human sputum: relathioship to inflammation. Scand J Clin Lab Invest 50: 509-516.

Cui FL, Fan J, Li JP, Hu Z (2004) Interactions between 1-benzoyl-4p-chlorophenyl thiosemicarbazide and serum albumin: Investigation by fluorescence spectroscopy. Bioorg Med Chem 12: 151-157.

Delaisse JM, Ledent P, Vaes G (1991) Collagenolytic cysteine proteinases of bone tissue. Cathepsin B, (pro)cathepsin L and a cathepsin L- like $70 \mathrm{kDa}$ proteinase. Biochem J 279: 167-174.

Ekiel I, Abrahamson M, Fulton D et al. (1997) NMR structural studies of human cystatin $\mathrm{C}$ dimers and monomers. I Mol Biol 271: 266277.

Feng XZ, Lin Z, Yang LJ, Wang C, Bai CL (1998) Investigation of the interaction between acridine orange and bovine serum albumin. Talanta 47: 1223-1229.

Gao H, Lei L, Liu J, Quin K, Chen X, Hu Z (2004) The study on the interaction between serum albumin and a new reagent with antitumour activity by spectrophotometric methods. I Photochem Photobiol 167: $213-221$. 
Hu YJ, Liu Y, Wang JB, Xiao XH, Qu SS (2004) Study of the interaction between monoammonium glycyrrhizinate and bovine serum albumin. J Pharm Biomed Anal 36: 915-919.

Hughes WT, Rivera G, Schell M et al. (1987) Successful intermittent chemoprophylaxis for Pneumocystis carinii pneumonitis. N Engl J Med 316: $1627-1632$.

Jensson O, Palsdottir A, Thorsteinsson L et al. (1990) Cystatin C mutation causing amyloid angiopathy and brain hemorrhage. Biol Chem Hoppe-Seyler 71: 229-232.

Kabanda A, Goffin E, Bernard A (1995) Factors influencing serum levels and peritoneal clearances of low molecular weight proteins in continuous ambulatory peritoneal dialysis. Kidney Int 48: 1946-1952.

Katzenstein AL, Myers JL (1998) Idiopathic pulmonary fibrosis: clinical relevance of pathologic classification. Am J Respir Crit Care Med 157: 1301-1315.

Khan MS, Bano B (2009) Purification, characterization and kinetics of thiol protease inhibitor from goat (Capra bircus) lung. Biochemistry (Moscow) 74: 781-788.

Kopper P, Baici A, Keist R et al (1994) Cathepsin B like proteinases as a marker for metastatic tumors cell variants. Exp Cell Biol 52:
293-299.

Kunitz M (1947) Crystalline soybean trypsin inhibitor: II. J Physiol 30: 291-310.

Neves C, Jorge R, Barcelos A (2009) The network of methotrexate toxicity. Acta Reumatol Port 34: 11-34.

North MJ, Moltram, JC, Coombs GH (1990) Cysteine proteinases of parasitic protozoa. Parasitol Today 6: 270-275.

Russell RE, Thorley A, Culpitt SV et al. (2002) Alveolar macrophagemediated elastolysis: roles of matrix metalloproteinases, cysteine, and serine proteases. Am J Physiol Lung Cell Mol Physiol 283: 867873 .
Scheiber J, Chen B, Milik M, Sukuru SCK, Bender A, Mikhailov D et al. (2009) Gaining insight into off-target mediated effects of drug candidates with a comprehensive systems chemical biology analysis. I Chem Inf Model 49: 308-317.

Shi GP, Sukhova GK, Grubb A, Ducharme A, Rhode LH, Lee RT, Ridker PM, Libby P, Chapman HA (1999) J Clin Invest 104: 11911197.

Sneppen K, Zocchi G (2005) Physics in molecular biology. Cambridge University Press, Cambridge, UK.

Takeda K, Wada A, Yamamoto K, Hachiya K, Batra PP (1998) Secondary structure change of myoglobin induced by sodium dodecyl sulfate and its kinetic aspects. J Colloid Interface Sci 125: 307-313.

Taniguchi CM, Armstrong SR, Green LC, Goaln DE, Tashjian AH Jr (2007) Fundamental principles of pharmacology. Chapter 5: pp 63-73. Wolters Kluwer, Lippincott Williams and Wilkins.

Trabant A, Gay RE, Gay RS (1991) Cathepsin B in synovial cell at the site of joint destruction in rheumatoid arthritis. Arthritis Rheum 34: 444.

Turk V, Stoka V, Turk D (2008) Cystatins: biochemical and structural properties, and medical relevance. Front Biosci 13: 5406-5420.

Wang Z, Zheng T, Zhu Z et al. (2000) Interferon gamma induction of pulmonary emphysema in the adult murine lung. J Exp Med 192: 1587-1600.

Surewicz WK, Mantsch HH, Chapman D (1993) Determination of protein secondary structure by Fourier-transform infrared spectroscopy: a critical assessment. Biochemistry 32: 389-394.

Zheng T, Zhu Z, Wang Z, Homer RJ, Ma B, Riese Jr RJ, Chapman Jr HA, Shapiro SD, Elias JA (2000) Inducible targeting of Il-13 to the adult lung causes matrix metalloproteinase-and cathepsin-dependent emphysema. J Clin Invest 106: 1081-1093. 